\title{
Piezoelectric Resonant Sensors with Contactless Interrogation for Mass-Sensitive and Acoustic-Load Detection
}

\author{
E. Tonoli *, M. Baù, M. Ferrari, V. Ferrari \\ Department of Information Engineering, University of Brescia, via Branze 38, 25123 Brescia (BS), Italy
}

\begin{abstract}
Piezoelectric resonator sensors that can be contactless interrogated as passive elements are proposed. The interrogation technique is based on time-gated excitation and detection phases, exploiting the sensing of the transient response of the resonator. The proposed system can be exploited for the measurement of physical or chemical quantities affecting the electromechanical resonant response. In particular, experimental results show the successful interrogation of variable-mass resonator sensors for humidity sensing in closed volumes and submersible resonator sensors operated in liquid environments.
\end{abstract}

(C) 2012 The Authors. Published by Elsevier Ltd. Selection and/or peer-review under responsibility of the Symposium Cracoviense Sp. z.o.o. Open access under CC BY-NC-ND license.

Keywords: Piezoelectric sensor; Contactless interrogation; Resonant sensor; Time-gated technique; Mass sensing; Acoustic load

\section{Introduction}

The contactless interrogation of passive sensors can be exploited in applications where cabled solutions are not allowed and environmental conditions are demanding. The resonant sensing principle represents a robust approach in contactless operation since it minimizes the detrimental influence of the interrogation distance on the readout signal. In this perspective, piezoelectric resonators are suitable to be used as contactless passive resonant sensors for the measurement of quantities affecting their resonant response. Quartz crystal resonators (QCR) and resonant piezo-layers (RPL) [1] are commonly employed as mass sensors in many bio-chemical applications [2] and contactless interrogation techniques have been reported for QCRs [3], though they typically require bare crystals or special-electrode sensors. We propose a timegated contactless interrogation technique and validate it on RPL sensors.

\footnotetext{
* Corresponding author. Tel.: +39-030-3715938; fax: +39-030-380014.

E-mail address: emanuele.tonoli@ing.unibs.it
} 


\section{Sensor structure and interrogation principle}

As shown in Fig. 1(a), the structure of the proposed RPL sensor is made of a lead zirconate titanate (PZT) layer, sandwiched between two electrode layers, screen-printed on alumina substrate. The PZT layer is poled along the thickness, Fig. 1(b) shows a RPL sensor, whose impedance spectrum, measured with a HP4194A impedance analyser around the fundamental resonance, is shown in Fig. 1(c).

The interrogation technique is based on a gated operation, somewhat similarly to what was previously proposed for silicon micromechanical resonators [4]. In this case, however, no additional magnets are required. As shown in Fig. 2, a primary coil $L_{1}$ is electromagnetically air-coupled to a secondary coil $L_{2}$ connected to the electrodes of the RPL. During the excitation phase the signal $v_{e}$ excites the RPL about its fundamental thickness-expansion resonance, while in the detection phase the excitation signal $v_{e}$ is turned off and the resonator undergoes decaying oscillations at frequency $f_{d m}$, which can be contactless sensed by measuring the voltage $v_{l}$ induced back across $L_{l}$. The voltage $v_{l}$ is then amplified and converted into a square waveform with frequency $f_{o}=f_{d m}$, which can be measured by a frequency counter.

The system has been modelled assuming a lumped-element approach, with the RPL modelled around its fundamental thickness-expansion resonance by adopting the Butterworth-van Dyke (BVD) circuit shown in Fig. 2. Accordingly, the induced voltage $v_{l}$ during the detection phase can be expressed as an exponentially damped sinusoid at frequency $f_{d m}$, which represents the RPL mechanical response. The mutual inductance $M$, which strongly depends on the interrogation distance $d$, virtually does not affect the RPL response parameters, thus making the principle robust against the interrogation distance.

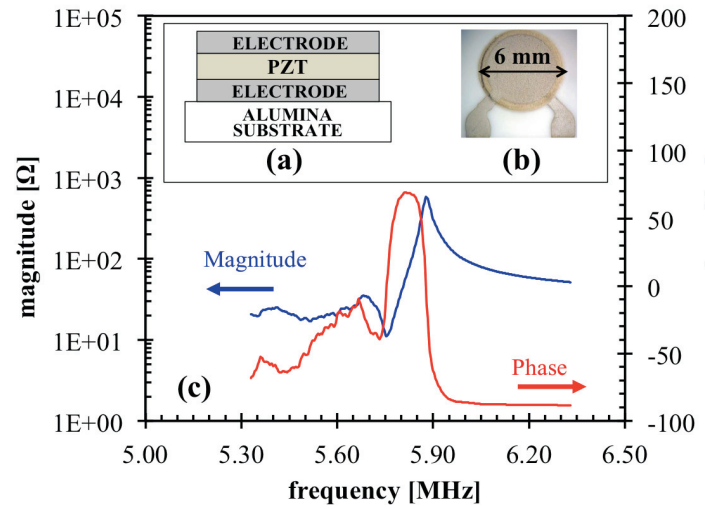

Fig. 1. (a) RPL structure, (b) picture of the manufactured device, and (c) measured impedance spectrum.

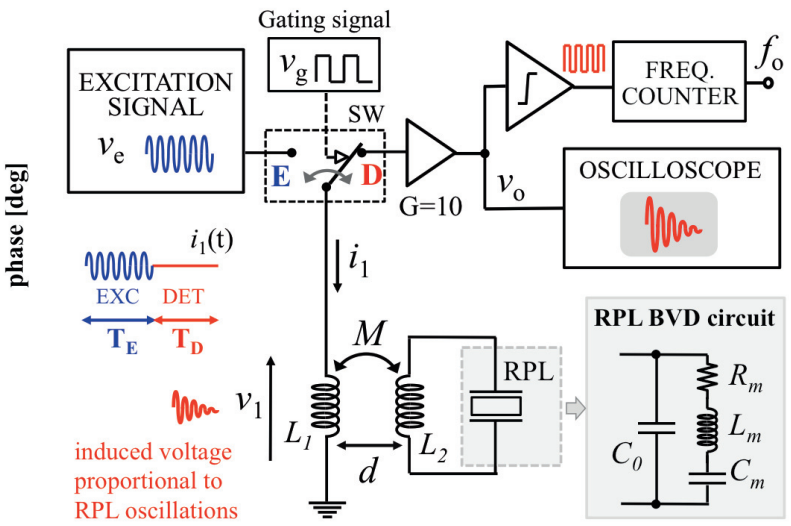

Fig. 2. Simplified diagram of the time-gated contactless interrogation system.

\section{Experimental results}

The principle has been applied to a $5.7 \mathrm{MHz}$ RPL sensor. The RPL BVD parameters are: $C_{0}=370 \mathrm{pF}$, $R_{m}=9.83 \Omega, L_{m}=48.09 \mu \mathrm{H}$ and $C_{m}=16.24 \mathrm{pF}$. Fig. 3 shows the typical readout voltage $v_{o}$ measured during the detection phase, with interrogation distance $d=15 \mathrm{~mm}$ and $L_{l}=L_{2}=8.5 \mu \mathrm{H}$. The frequency $f_{e}$ and amplitude of the excitation signal $v_{e}$ have been set to $5.9 \mathrm{MHz}$ and $5 \mathrm{~V}$, respectively. As predicted by the theoretical model, the RPL mechanically responds with a damped sinusoidal signal. From the envelope a damped resonant frequency $f_{o}=f_{d m}=5.893 \mathrm{MHz}$ and a quality factor $Q=160$ can be estimated. These values are in good agreement with the predictions of the model, which are $f_{d m}=5.856 \mathrm{MHz}$ and $Q=174$. 
Fig. 4 shows the readout frequency $f_{o}$ and signal amplitude $v_{o}$ versus the interrogation distance $d$. Typical values for $d$ are in the order of $2 \mathrm{~cm}$ and, as predicted by the model, it does not affect the measured readout frequency, acting only as scaling factor on the amplitude of the readout voltage.

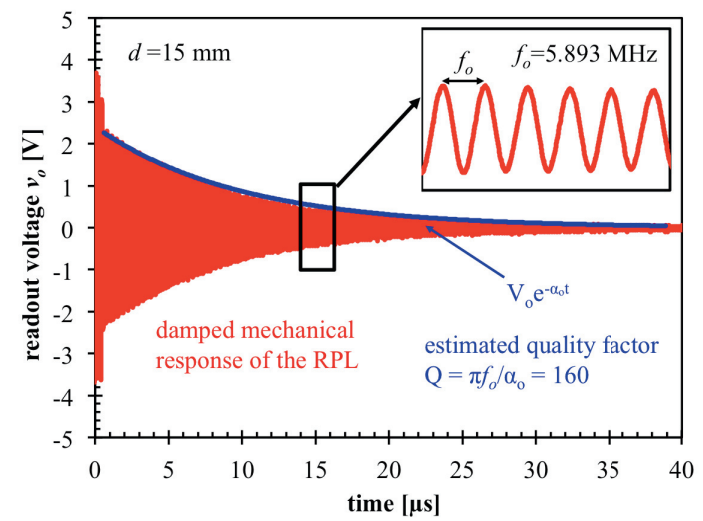

Fig. 3. Response of a 5.7 MHz RPL during the detection phase.

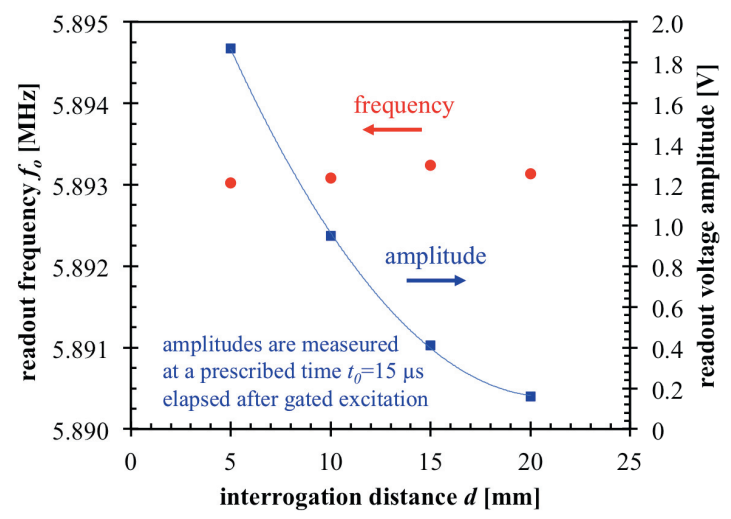

Fig. 4. Measured readout frequency $f_{o}$ and readout voltage amplitude for different values of the interrogation distance $d$.

The interrogation principle has been used to perform contactless gravimetric humidity measurements using a $6.5 \mathrm{MHz}$ RPL coated with Polyvinylpyrrolidone (PVP) as sensing film. The BVD parameters of the coated resonator at room atmosphere are: $C_{0}=1.02 \mathrm{nF}, R_{m}=9.95 \Omega, L_{m}=5.94 \mu \mathrm{H}$ and $C_{m}=104.64 \mathrm{pF}$. The block diagram of the measurement setup is shown in Fig. 5 [5]. The RPL sensor, connected to the secondary coil, has been placed inside a stainless steel chamber with a Teflon bottom side. The relative humidity $(\mathrm{RH})$ inside the chamber has been varied with a computer-driven system and monitored by a $\mathrm{RH}$ sensor (Gefran S6000) used as a reference. The measurements have been performed with a temperature inside the sensor chamber of $26^{\circ} \mathrm{C} \pm 0.5^{\circ} \mathrm{C}$, with the interrogation distance $d=2 \mathrm{~cm}$ and $L_{l}=L_{2}=8.5 \mu \mathrm{H}$. Fig. 6 shows the measured response of the RPL sensor versus time, compared with the RH inside the sensor chamber. The measured frequency shift $\Delta f_{o}$ follows the RH trend, thus demonstrating the capability of the developed system to contactless interrogate the sensor from outside the chamber.

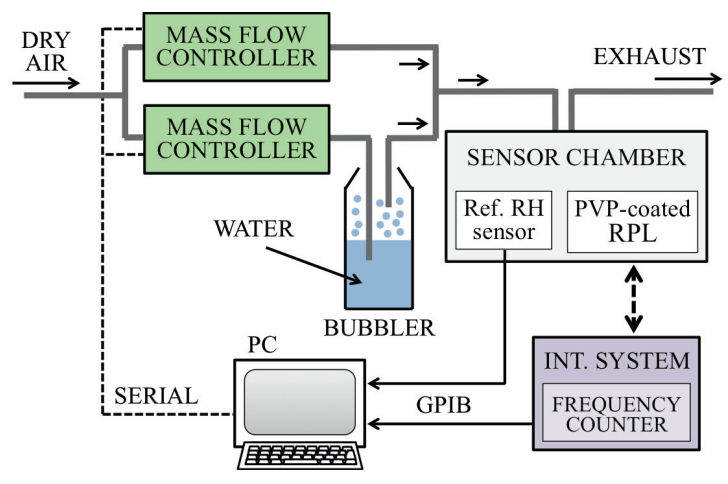

Fig. 5. Block diagram of the humidity measurement system.

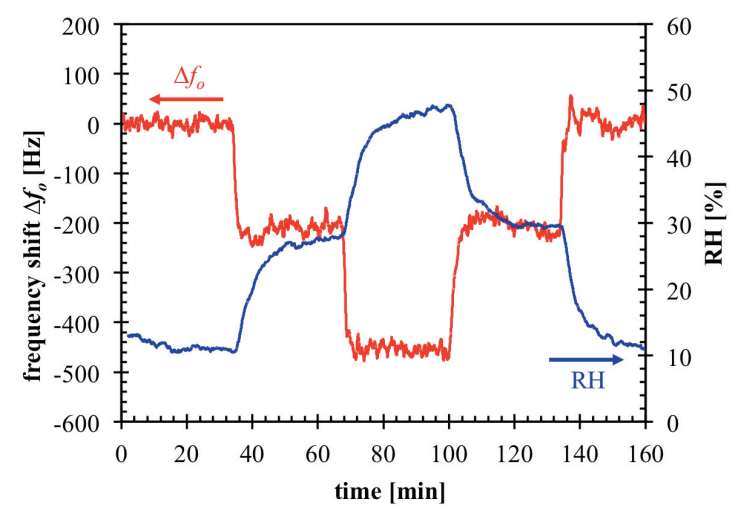

Fig. 6. Contactless RH measurements with a PVP-coated RPL.

The 5.7 MHz RPL and the secondary coil $L_{2}=3.2 \mu \mathrm{H}$ have been arranged together composing a sealed submersible sensor capsule, as shown in Fig. 7, which has been used to perform contactless interrogation 
with the sensor operated in liquid environments. Fig. 8 shows the response of the RPL sensor operated in water and ethanol. In the liquid-filled cell with horizontally mounted RPL, the air/liquid interface acts as a reflection surface for the compressional waves produces by the RPL, generating a standing wave pattern. The liquid evaporation results in periodic changes in the readout frequency $f_{o}$. The periodicities are $T_{w}=\lambda_{w} / 2 k_{w}$ in water and $T_{e}=\lambda_{e} / 2 k_{e}$ in ethanol, with $k_{w}$ and $k_{e}$ representing the liquid evaporation rates expressed in $\mathrm{m} / \mathrm{s}$, and $\lambda_{w}$ and $\lambda_{e}$ representing the wavelength of the compressional wave in the two liquids.

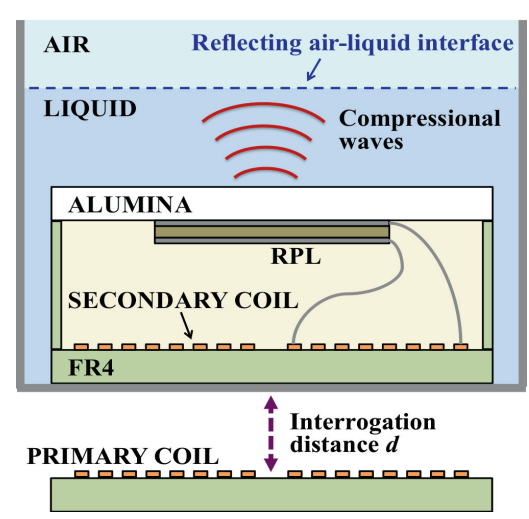

Fig. 7. Apparatus used for the 5.7 MHz RPL submersible sensor capsule operated in liquid environments.

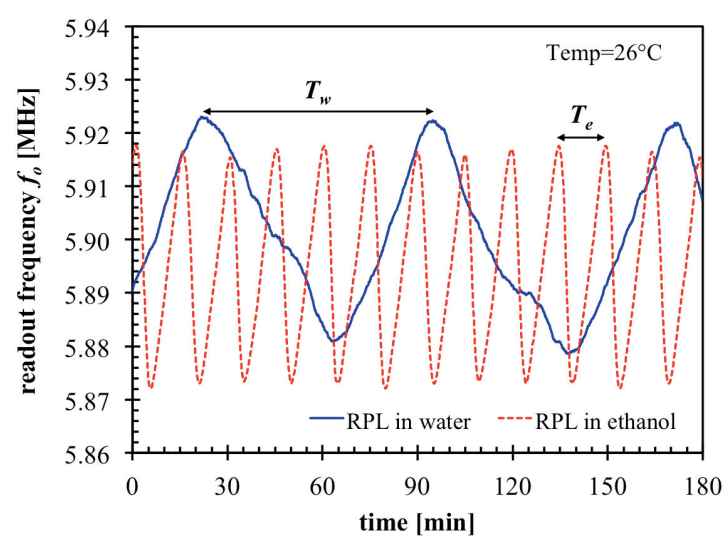

Fig. 8. Measured response of the RPL sensor operated in water and ethanol.

\section{Conclusions}

Piezoelectric resonator sensors based on PZT thick films that can be contactless interrogated as passive elements have been proposed. The developed interrogation technique is based on the time separation of the excitation and detection phases, exploiting the sensing of the resonator transient response. The principle has been studied by means of a lumped-element equivalent circuit. Experimental results validate the theoretical model and show the successful contactless interrogation of the proposed sensors for humidity sensing in closed volumes and in a submersible sensor capsule operated in liquid. The interrogation technique is exploitable for chemico-physical measurements of quantities affecting the sensor resonant response.

\section{References}

[1] Ferrari V, Marioli D, Taroni A, Theory, modelling and characterization of PZT-on-alumina resonant piezo-layers as acoustic-wave mass sensors, Sensors and Actuators A 2001, 92:182-190

[2] Janshoff A., Steinem C., Quartz Crystal Microbalance for Bioanalytical Applications, Sensors Update 2001, 9:313-354

[3] Ogi H, Motoshisa K, Matsumoto T, Hatanaka K, Hirao M, Isolated electrodeless high-frequency quartz crystal microbalance for immunosensors, Analytical Chemistry 2006, 78:6903-6909

[4] Baù M, Tonoli E, Ferrari V, Marioli D, Contactless electromagnetic switched interrogation of micromechanical cantilever resonators, Sensors and Actuators A 2011, 172:195-203

[5] Ferrari M, Ferrari V, Marioli D, Taroni A, Suman M, Dalcanale E, Cavitand-coated PZT resonant piezo-layer sensors: properties, structure, and comparison with QCM sensors at different temperatures under exposure to organic vapors, Sensors and Actuators B 2004, 103:240-246 